\title{
Multilevel coherences in quantum dots
}

\author{
Martin T. Maurer $\odot,{ }^{1}$ Jürgen König $\odot,{ }^{2}$ and Herbert Schoeller ${ }^{1}$ \\ ${ }^{1}$ Institut für Theorie der Statistischen Physik, RWTH Aachen, 52056 Aachen, Germany \\ and JARA-Fundamentals of Future Information Technology \\ ${ }^{2}$ Theoretische Physik and CENIDE, Universität Duisburg-Essen, 47048 Duisburg, Germany
}

(Received 12 April 2020; revised 26 August 2020; accepted 27 August 2020; published 18 September 2020)

\begin{abstract}
We study transport through strongly interacting quantum dots with $N$ energy levels that are weakly coupled to generic multichannel metallic leads. In the regime of coherent sequential tunneling, where level spacing and broadening are of the same order but small compared to temperature, we present a unified SU $(N)$-invariant form of the kinetic equation for the reduced density matrix of the dot and the tunneling current. This is achieved by introducing the concept of flavor polarization for the dot and the reservoirs and splitting the kinetic equation in terms of flavor accumulation, anisotropic flavor relaxation, and exchange-field- and detuning-induced flavor rotation. In particular, we identify the exchange field as the cause of negative differential conductance at offresonance bias voltages appearing in generic quantum-dot models. To illustrate the notion of flavor polarization, we analyze the nonlinear current through a triple-quantum-dot device.
\end{abstract}

DOI: 10.1103/PhysRevResearch.2.033440

\section{INTRODUCTION}

The spatial confinement of electrons in quantum dots gives rise to both a charging energy and a discrete spectrum of single-particle energy levels. If two or more levels are energetically close to each other compared to their tunnelinginduced broadening, coherent superpositions may form and influence the electronic transport through the quantum dots. By coupling a spin- $\frac{1}{2}$ dot level to ferromagnetic leads (thereby forming a quantum-dot spin valve) and applying a bias voltage, the interplay of spin accumulation, relaxation, and precession gives rise to a nonequilibrium polarization of the quantum-dot spin [1-11]. Controlling transport by generating and manipulating spins is the declared goal of the field of spintronics.

The SU(2) framework for the spin degree of freedom is easily transferred to other two-level systems by introducing an isospin. This includes the valley degree of freedom in the band structure of graphene and carbon nanotubes, studied in the field of valleytronics [12,13]. Another example is given by quantum-dot Aharonov-Bohm interferometers, in which the coherent superposition of the orbital levels of two single-level quantum dots gives rise to Aharonov-Bohm oscillations of the current through the device [14-16]. Furthermore, superconducting correlations in quantum dots attached to superconducting leads have been described in terms of an isospin defined by two quantum-dot states with different particle numbers [17].

Published by the American Physical Society under the terms of the Creative Commons Attribution 4.0 International license. Further distribution of this work must maintain attribution to the author(s) and the published article's title, journal citation, and DOI.
In recent decades, triple quantum dots have been realized experimentally [18-21]. In such structures, three instead of two states can be energetically close to each other, suggesting an SU(3) framework. Even coherences between more than three levels are realized in molecules such as benzene [22,23]. Common among these systems are coherence-induced transport signatures such as negative differential conductance (NDC) and complete current blockades, making them interesting for technological application in nanoelectronic devices. It is therefore of high interest to find a description of the complex nonequilibrium behavior of generic $N$-level dots in a unified and physically intuitive way similar to spin-valve systems.

In this paper, we seek such a description for quantum dots with an arbitrary number $N$ of orbitals coupled to generic multichannel metallic leads. The underlying group in this case is $\mathrm{SU}(N)$. We will present a unified theoretical framework for the regime where the level spacing $\Delta$ and the broadening $\Gamma$ are of the same order and small compared to temperature $T$, which we refer to as the coherent-sequential-tunneling regime. It is of particular interest since it exhibits quantum coherence in weak coupling and is most easily accessible to experiments. Similarly to quantum-optics approaches [24], we represent the density matrix of the dot by a real vector, which we refer to as the flavor polarization of the dot. In addition, we define also a set of flavor polarizations for the reservoirs, which is crucial to understand the NDC physics induced by quantum coherence. We show that the kinetic equations governing the dot dynamics can be cast in a universal $\mathrm{SU}(N)$-invariant form containing terms that describe dot-flavor accumulation, relaxation, and rotation, suggesting the term flavortronics to describe transport through $N$-level quantum dots. A central result of our work is the identification of flavor rotations as the generic cause of NDC at off-resonance bias voltages. We illustrate this and the general 
usefulness of the flavor-polarization formalism by analyzing the $I-V$ characteristic of a triple-dot setup.

\section{MODEL}

We consider $N$ spinless quantum-dot orbitals with strong Coulomb interaction that are weakly coupled to multichannel metallic leads. The total Hamiltonian is given by $H=$ $H_{\mathrm{D}}+H_{\text {res }}+H_{\mathrm{T}}$. For convenience, we work in a basis where the single-particle part of the dot Hamiltonian is already diagonalized. Including the interaction, the dot is described by $H_{\mathrm{D}}=H_{\mathrm{D}}^{0}+H_{\mathrm{D}}^{\mathrm{int}}=\sum_{i=1}^{N} \epsilon_{i} c_{i}^{\dagger} c_{i}+U \sum_{i<i^{\prime}} c_{i}^{\dagger} c_{i} c_{i^{\prime}}^{\dagger} c_{i^{\prime}}$. The average level position is defined by $\epsilon=\sum_{i=1}^{N} \epsilon_{i} / N$ and the detunings by $\Delta_{i j}=\epsilon_{i}-\epsilon_{j}$. For large Coulomb interaction $U \rightarrow \infty$, only the empty and the singly occupied dot configurations are allowed. The leads $H_{\text {res }}=\sum_{r=1}^{N_{\text {res }}} H_{r}$ with $H_{r}=$ $\sum_{k \nu} \epsilon_{r k \nu} a_{r k \nu}^{\dagger} a_{r k \nu}$ are modeled as reservoirs of noninteracting electrons with temperature $T$ and chemical potential $\mu_{r}$. The channel index $v=1, \ldots, N_{\mathrm{ch}}$ accounts for different bands and the quantum number $k$ labels the energy eigenstates in each band. The reservoir density of states $\rho_{r}(\omega)=$ $\rho_{0} \Lambda^{2} /\left[\left(\omega-\mu_{r}\right)^{2}+\Lambda^{2}\right]$ contains a high-energy cutoff $\Lambda$ ensuring convergence of appearing integrals. Tunneling between dot and leads is described by $H_{\mathrm{T}}=\sum_{r v i} t_{v i}^{r} a_{r k v}^{\dagger} c_{i}+$ H.c., with energy-independent tunneling amplitudes $t_{v i}^{r}$. The latter enter the $N \times N$ Hermitian, positive-semidefinite hybridization matrices $\Upsilon^{r}$ with matrix elements $\Upsilon_{i j}^{r}=2 \pi \rho_{0} \sum_{v}\left(t_{v i}^{r}\right)^{*} t_{v j}^{r}$. The tunnel-coupling strength to reservoir $r$ is characterized by $\Gamma^{r}=\operatorname{Tr} \Upsilon^{r} / N$ and the total tunneling strength by $\Gamma=\sum_{r} \Gamma^{r}$. We set $e=\hbar=k_{B}=1$ throughout this paper.

\section{FLAVOR REPRESENTATION OF THE QUANTUM-DOT STATE}

Since the infinite charging energy limits the number $N_{e}$ of electrons in the quantum dot to 0 and 1, the Hilbert space of the quantum-dot states is $N+1$ dimensional with basis states $|0\rangle$ for an empty quantum dot and $|i\rangle$ for an electron occupying level $i=1,2, \ldots, N$. As a result, the reduced density matrix $\rho=\rho_{N_{e}=0}+\rho_{N_{e}=1}$ of the quantum dot can be decomposed into a part $\rho_{N_{e}=0}=P_{0}|0\rangle\langle 0|$ describing the empty quantum dot (with probability $P_{0}$ ) and a part $\rho_{N_{e}=1}$ for single occupation (with probability $P_{1}=\operatorname{Tr} \rho_{N_{e}=1}=1-P_{0}$ ). The latter is an $N \times N$ Hermitian positive-semidefinite matrix that can be decomposed into the identity matrix $\mathbb{1}_{N}$ and a set of $s_{N}=$ $N^{2}-1$ traceless generators $\left\{\lambda_{a}\right\}$ of $\mathrm{SU}(N)$, which are normalized such that $\operatorname{Tr}\left(\lambda_{a} \lambda_{b}\right)=2 \delta_{a b},\left[\lambda_{a}, \lambda_{b}\right]_{-}=2 i \sum_{c} f_{a b c} \lambda_{c}$, and $\left[\lambda_{a}, \lambda_{b}\right]_{+}=\frac{4}{N} \delta_{a b}+2 \sum_{c} d_{a b c} \lambda_{c}$, with real constants $f_{a b c}$ and $d_{a b c}$ forming a totally antisymmetric and a symmetric tensor, respectively. ${ }^{1}$ As a result $[26,27]$, the density matrix for single occupation

$$
\rho_{N_{e}=1}=\frac{1}{N}\left(P_{1} \mathbb{1}_{N}+c_{N} \boldsymbol{g} \cdot \boldsymbol{\lambda}\right),
$$

\footnotetext{
${ }^{1}$ The numerical values of these constants depend on the chosen set of generators. A straightforward choice is the generalized Gell-Mann matrices [25].
}

with $\boldsymbol{g} \cdot \boldsymbol{\lambda}=\sum_{a} g_{a} \lambda_{a} \quad$ and $\quad c_{N}=\sqrt{N(N-1) / 2}, \quad$ is parametrized by the probability $P_{1}$ of single occupation and the components $g_{a}$ of an $s_{N}$-dimensional real vector $\boldsymbol{g}$, referred to as flavor polarization of the dot. Semipositivity of $\rho_{N_{e}=1}$ implies $\operatorname{Tr} \rho_{N_{e}=1}^{2} \leqslant P_{1}^{2}$, which yields $|\boldsymbol{g}| \leqslant P_{1}$, i.e., the normalization is chosen such that $|\boldsymbol{g}|=1$ describes maximal flavor polarization. The $s_{N}$-dimensional flavor-polarization vector $\boldsymbol{g}$ generalizes the three-dimensional spin-polarization vector in the case of a spinful quantum-dot level for $N=2$ to any number $N$ of quantum dot levels. We note that for $N>2$, flavor polarization is fundamentally different from angular momentum $(N-1) / 2$, as the latter is described in terms of the $N$-dimensional representation of the three generators of $\mathrm{SU}(2)$ and not of the $s_{N}$ generators of $\mathrm{SU}(N)$.

The dot flavor polarization carries the information about the mixture and superpositions of dot states contained in the density matrix. The modulus $|\boldsymbol{g}|$ is a measure for the purity in the one-particle sector, defined as $\gamma=\operatorname{Tr}\left[\left(\rho_{N_{e}=1} / P_{1}\right)^{2}\right]=$ $\left[1+(N-1)\left(|\boldsymbol{g}| / P_{1}\right)^{2}\right] / N .^{2}$

Thus, maximal flavor polarization $\left|\boldsymbol{g}_{1}\right|=1$ corresponds to a pure state in which, in a properly chosen basis, one of the $N$ dot levels is occupied with probability 1 . All mixed or pure states with this specific dot level being empty are described by flavor-polarization vectors $g_{2}$ that satisfy the condition $g_{1}$. $\boldsymbol{g}_{2}=-1 /(N-1)$. In contrast, vanishing flavor polarization corresponds to the maximally mixed state.

The notion of an $s_{N}$-dimensional flavor polarization vector is needed not only for the dot but also for each reservoir. The reservoir flavor polarization $\boldsymbol{n}^{r}$ (with $\left|\boldsymbol{n}^{r}\right| \leqslant 1$ ) is defined by the decomposition

$$
\Upsilon^{r}=\Gamma^{r}\left(\mathbb{1}_{N}+c_{N} \boldsymbol{n}^{r} \cdot \lambda\right)
$$

of the hybridization matrix, i.e., $\Gamma^{r}$ and $\boldsymbol{n}^{r}$ contain all microscopic details of the tunnel coupling. Full polarization $\left|\boldsymbol{n}^{r}\right|=$ 1 occurs when all channels couple to the same dot state, while vanishing polarization $\boldsymbol{n}^{r}=0$ corresponds to $N$ channels that are coupled with equal strength to a different one of the $N$ dot levels each.

To determine the components of $\boldsymbol{g}$ and $\boldsymbol{n}^{r}$ for given density and hybridization matrices, we make use of the orthogonality of the generators to arrive at $g_{a}=N \operatorname{Tr}\left(\rho_{N_{e}=1} \lambda_{a}\right) / 2 c_{N}$ and $\Gamma^{r} n_{a}^{r}=\operatorname{Tr}\left(\Upsilon^{r} \lambda_{a}\right) / 2 c_{N}$. Finally, we remark that only a subset of the vectors $\boldsymbol{g}$ or $\boldsymbol{n}^{r}$ in the $s_{N}$-dimensional unit sphere describes flavor polarization, i.e., corresponds to a (positivesemidefinite) density or hybridization matrix [26-28].

\section{KINETIC EQUATION}

The quantum-dot state, including its flavor polarization, is described by the reduced density matrix $\rho$ with matrix elements $\rho_{\chi \chi^{\prime}}=\left\langle\mid \chi^{\prime}\right\rangle\langle\chi \mid\rangle$. The natural basis states $|\chi\rangle$ are the empty dot $|0\rangle$ and single occupation $|i\rangle$ of level $i=$ $1,2, \ldots, N$. The diagonal entries $\rho_{\chi \chi}$ are the probabilities to find the dot in state $|\chi\rangle$, while the off-diagonals $\rho_{i j}$ describe coherences between levels $i$ and $j$. In the weak-coupling and

\footnotetext{
${ }^{2}$ The scaling by $P_{1}$ in this definition ensures that the purity takes the usual values $\gamma \in[1 / N, 1]$.
} 
Markov regime $t^{-1}, \Gamma \ll T$, the kinetic equations of $\rho_{\chi \chi^{\prime}}$ read

$$
\frac{d}{d t} \rho_{\chi x^{\prime}}=-i\left(\epsilon_{\chi}-\epsilon_{\chi^{\prime}}\right) \rho_{\chi \chi^{\prime}}+\sum_{\eta \eta^{\prime}} W_{\chi \chi^{\prime}, \eta \eta^{\prime}} \rho_{\eta \eta^{\prime}} .
$$

The generalized transition matrix elements $W_{\chi x^{\prime}, \eta \eta^{\prime}}$ in Liouville space, represented as irreducible diagrams on the Keldysh contour, are calculated up to first order in $\Gamma$ employing a real-time diagrammatic technique presented in $[29,30]$ (see Appendix A for details). The current $I_{r}$ from the dot into reservoir $r$ can then be calculated from $\rho$ and a partial selection of diagrams.

In the coherent-sequential-tunneling regime $\left|\Delta_{i j}\right| \lesssim \Gamma$, we express the kinetic equations in terms of the flavor polarization in a coordinate-free form that makes the $\mathrm{SU}(N)$ invariance explicit (see Appendix D). This is done by reading (3) as a matrix equation, inserting the flavor decompositions (1) and (2) for each appearing density and hybridization matrix, and using the relations $P_{1}=\sum_{i=1}^{N} \rho_{i i}$ and $g_{a}=N \operatorname{Tr}\left(\rho_{N_{e}=1} \lambda_{a}\right) / 2 c_{N}$. We find

$$
\frac{d P_{1}}{d t}=\sum_{r} \Gamma^{r}\left\{N f_{r}^{+}(\epsilon) P_{0}-f_{r}^{-}(\epsilon)\left[P_{1}+(N-1) \boldsymbol{n}^{r} \cdot g\right]\right\}
$$

for the total-occupation number and

$$
\begin{aligned}
\frac{d \boldsymbol{g}}{d t} & =\left(\frac{d \boldsymbol{g}}{d t}\right)_{\mathrm{acc}}+\left(\frac{d \boldsymbol{g}}{d t}\right)_{\mathrm{rel}}+\left(\frac{d \boldsymbol{g}}{d t}\right)_{\mathrm{rot}}, \\
\left(\frac{d \boldsymbol{g}}{d t}\right)_{\mathrm{acc}} & =\sum_{r} \Gamma^{r}\left[N f_{r}^{+}(\epsilon) P_{0}-f_{r}^{-}(\epsilon) P_{1}\right] \boldsymbol{n}^{r}, \\
\left(\frac{d \boldsymbol{g}}{d t}\right)_{\mathrm{rel}} & =-\sum_{r} \Gamma^{r} f_{r}^{-}(\epsilon)\left(\boldsymbol{g}+\boldsymbol{n}^{r} * \boldsymbol{g}\right), \\
\left(\frac{d \boldsymbol{g}}{d t}\right)_{\mathrm{rot}} & =\boldsymbol{B}_{\mathrm{tot}} \wedge \boldsymbol{g}
\end{aligned}
$$

for the flavor polarization. Here $f_{r}^{+}(\epsilon)=1 /\{\exp [\beta(\epsilon-$ $\left.\left.\left.\mu_{r}\right)\right]+1\right\}$ is the Fermi function with $\beta=1 / T, f_{r}^{-}(\epsilon)=1-$ $f_{r}^{+}(\epsilon), \boldsymbol{B}_{\mathrm{tot}}=\boldsymbol{B}+\boldsymbol{B}_{\mathrm{ex}}, \boldsymbol{B}=\operatorname{Tr}\left(H_{\mathrm{D}}^{0} \lambda\right) / c_{N}, \boldsymbol{B}_{\mathrm{ex}}=\sum_{r} \boldsymbol{B}_{\mathrm{ex}}^{r}$, and

$$
\boldsymbol{B}_{\mathrm{ex}}^{r}=\frac{\Gamma^{r}}{\pi}\left[\operatorname{Re} \psi\left(\frac{\pi+i \beta\left(\mu_{r}-\epsilon\right)}{2 \pi}\right)-\psi\left(\frac{\pi+\beta \Lambda}{2 \pi}\right)\right] \boldsymbol{n}^{r},
$$

with the digamma function $\psi$. The star and wedge products $(\boldsymbol{x} * \boldsymbol{y})_{a}=c_{N} \sum_{b c} d_{a b c} x_{b} y_{c}$ and $(\boldsymbol{x} \wedge \boldsymbol{y})_{a}=c_{N} \sum_{b c} f_{a b c} x_{b} y_{c}$ are straightforward generalizations of those defined for the $\mathrm{SU}(3)$ case in [31] and respect the $\mathrm{SU}(N)$ invariance. The equation for $P_{0}$ follows simply from $d P_{0} / d t=-d P_{1} / d t$.

The kinetic equations essentially generalize those for the spin in a quantum-dot spin valve [2] to arbitrary flavor number $N$. The equations show that dot occupation $P_{1}$ and flavor polarization $\boldsymbol{g}$ are coupled. The scalar product $\boldsymbol{n}^{r} \cdot \boldsymbol{g}$ reflects how strongly the dot electron couples to reservoir $r$. This affects the rate of tunneling processes from the dot into $r$ [see Eq. (4)].

We have split the equation for $d g / d t$ into three parts. The first part (6) describes flavor accumulation due to tunneling between dot and flavor-polarized reservoirs. For each reservoir $r$, the contribution to flavor accumulation is proportional to $\boldsymbol{n}^{r}$.
The second term (7) describes flavor relaxation. It can be written as $(d \boldsymbol{g} / d t)_{\mathrm{rel}}=-\sum_{r} \Gamma^{r} f_{r}^{-}(\epsilon) D^{r} \boldsymbol{g}$ by introducing the matrix $D^{r}$ with matrix elements $D_{a c}^{r}=\delta_{a c}+c_{N} \sum_{b} d_{a b c} n_{b}^{r}$. Because $D^{r}$ is positive semidefinite (see Appendix C), the relaxation term always reduces the modulus of the flavor polarization $(d|\boldsymbol{g}| / d t)_{\text {rel }} \leqslant 0$. The matrix $D^{r}$ differs from the identity matrix, which makes flavor relaxation anisotropic. ${ }^{3}$

The last term (8) describes flavor rotation. It can be rewritten as $(d \boldsymbol{g} / d t)_{\mathrm{rot}}=F \boldsymbol{g}$ by introducing the matrix $F$ with matrix elements $F_{a c}=c_{N} \sum_{b} f_{a b c} B_{\mathrm{tot}, b}$. Due to $f_{a b c}=$ $-f_{c b a}, F$ is skew symmetric and therefore generates an $s_{N}$-dimensional rotation. ${ }^{4}$ Two mechanisms lead to flavor rotation. The detuning-induced part $\boldsymbol{B}$ generalizes the Zeeman-field induced spin rotation in the SU(2) case. The contribution $\boldsymbol{B}_{\mathrm{ex}}$ is induced by virtual tunneling of quantumdot electrons into the flavor-polarized reservoirs and back. We call $\boldsymbol{B}_{\text {ex }}$ an exchange field, in analogy to the one leading to Larmor precession of the spin in quantum-dot spin valves $[1,2]$. Besides its dependence on the reservoir flavor polarizations, its magnitude can be controlled via bias voltage, level positions, and coupling strengths [see Eq. (9)]. As the term in large square brackets in Eq. (9) is nonzero in the wideband limit of large $\Lambda$, the individual reservoir exchange fields are nonzero for polarized reservoirs and the total exchange field $\boldsymbol{B}_{\text {ex }}$ can only vanish in highly symmetric setups where different $\boldsymbol{B}_{\mathrm{ex}}^{r}$ cancel.

The flavor polarization affects transport through the quantum dot. In the coherent-sequential-tunneling regime $\left|\Delta_{i j}\right| \lesssim$ $\Gamma$, the current into reservoir $r$ is

$$
I_{r}=\Gamma^{r}\left\{-N f_{r}^{+}(\epsilon) P_{0}+f_{r}^{-}(\epsilon)\left[P_{1}+(N-1) \boldsymbol{n}^{r} \cdot \boldsymbol{g}\right]\right\} .
$$

In the special case of a singly occupied $\operatorname{dot} P_{1}=1$ and a flavor polarization $\boldsymbol{g}$ satisfying $\boldsymbol{n}^{r} \cdot \boldsymbol{g}=-1 /(N-1)$, no current flows into the reservoir. This flavor blockade appears since the states corresponding to $\boldsymbol{g}$ decouple from the reservoir.

The kinetic equations (4)-(8) and the current formula (10) are the main results of our paper. They provide an intuitive picture of the dot dynamics and the electronic transport in terms of the flavor polarization. We emphasize the special role of the exchange field (9). Its dependence on the chemical potentials is responsible for the NDC at off-resonance bias voltages, where all Fermi functions are constant. The precise mechanism is discussed below for the simple example of a triple quantum dot, but the same reasoning applies to any setup with $N$ levels in the coherent-sequential-tunneling regime. While current blockades due to coherence effects and resulting NDC have been widely studied [32-43], this intuitive explanation of off-resonance NDC for generic $N$-level setups closes a gap in the literature.

In the opposite incoherent-sequential-tunneling regime of large detunings $\left|\Delta_{i j}\right| \sim T \gg \Gamma$, the coherences can be neglected and both contributions to the rotation term drop out. In that case, the kinetic equations simplify to the standard Fermi

\footnotetext{
${ }^{3}$ An exception is the $\mathrm{SU}(2)$ case, where the $d$ 's are vanishing, which makes spin relaxation in quantum-dot spin valves isotropic [2].

${ }^{4}$ Similar rotations of coherence vectors have been discussed in the context of quantum optics [25] and open quantum systems in general [24].
} 


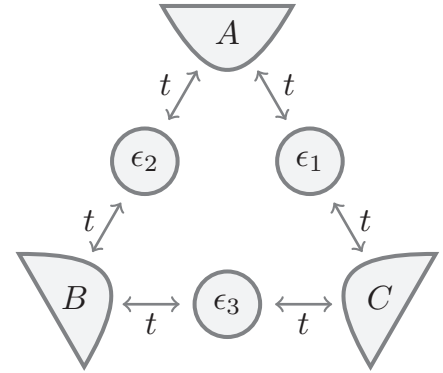

FIG. 1. Three reservoirs are coupled to two levels each. Nonzero tunnel couplings are chosen real and equal. A bias voltage $V$ is applied such that $\mu_{B}=\mu_{C}=-\mu_{A}=V / 2$.

golden rule rate equations $d \rho_{i i} / d t=\sum_{r}\left[\Upsilon_{i i}^{r} f_{r}^{+}\left(\epsilon_{i}\right) \rho_{00}-\right.$ $\left.\Upsilon_{i i}^{r} f_{r}^{-}\left(\epsilon_{i}\right) \rho_{i i}\right]$ and $d \rho_{00} / d t=-\sum_{i=1}^{N} d \rho_{i i} / d t$, as well as $I_{r}=$ $\sum_{i}\left[-\Upsilon_{i i}^{r} f_{r}^{+}\left(\epsilon_{i}\right) \rho_{00}+\Upsilon_{i i}^{r} f_{r}^{-}\left(\epsilon_{i}\right) \rho_{i i}\right]$.

Let us briefly consider the general case, where the levels are arranged in multiple groups of close-lying energies. This case can be treated straightforwardly by the formalism. All isolated levels enter the master equations via the Fermi golden rule rate equations. Regarding groups of at least two closelying levels, flavor equations must be set up for each group, defining adequate flavor polarizations from the projections of the density and hybridization matrices onto the subspace of states included in the group.

Finally, we remark that an additional spin degeneracy of the quantum-dot levels can be easily taken into account without doubling $N$. All presented formulas remain valid once $P_{0}=\rho_{00}$ appearing on the right-hand side is multiplied by a factor of 2 , while $\rho_{i j}$ is understood as $\sum_{\sigma} \rho_{i \sigma, j \sigma}$, i.e., spin affects the results only quantitatively. In the following example, we assume spinless electrons.

\section{EXAMPLE}

We illustrate the usefulness of the concept of flavor polarization by analyzing the current through the triple-dot setup shown in Fig. 1. Each of the three reservoirs $r=A, B, C$ couples symmetrically to two dot levels, such that $\Gamma^{r}=\Gamma / 3$, and accommodates one channel only, which implies maximal flavor polarization $\left(\left|\boldsymbol{n}^{r}\right|=1\right)$. We choose the standard Gell-Mann matrices [44] (see Appendix E for a list) as the generators of $\mathrm{SU}(3)$. Then the explicit flavor-polarization vectors are given by $\boldsymbol{n}^{A}=(\sqrt{3} / 2,0,0,0,0,0,0,1 / 2)$, $\boldsymbol{n}^{B}=(0,0,-\sqrt{3} / 4,0,0, \sqrt{3} / 2,0,-1 / 4), \quad$ and $\quad \boldsymbol{n}^{C}=$ $(0,0, \sqrt{3} / 4, \sqrt{3} / 2,0,0,0,-1 / 4)$. The chemical potentials are set to $\mu_{B}=\mu_{C}=-\mu_{A}=V / 2$, i.e., leads $B$ and $C$ can be combined into a single lead $B C$ with flavor polarization $\boldsymbol{n}^{B C}=\left(\boldsymbol{n}^{B}+\boldsymbol{n}^{C}\right) / 2$ and coupling strength $\Gamma^{B C}=2 \Gamma / 3$. Using the flavor framework, we will be able to explain NDC and current blockades due to coherence effects (similar as reported in Refs. [32-43]) in terms of flavor blockade and its lifting by flavor rotation.

In Fig. 2 we show the current into reservoir $A$ for an average dot-level energy of $\epsilon=\epsilon_{3}=25 \Gamma$ and symmetric detunings $\epsilon_{1 / 2}=\epsilon \pm \Delta$ as a function of bias voltage $V$. We find the expected increase in current as the chemical potentials approach the dot level energies. At higher voltages, the current exhibits signatures of quantum coherence for detunings of

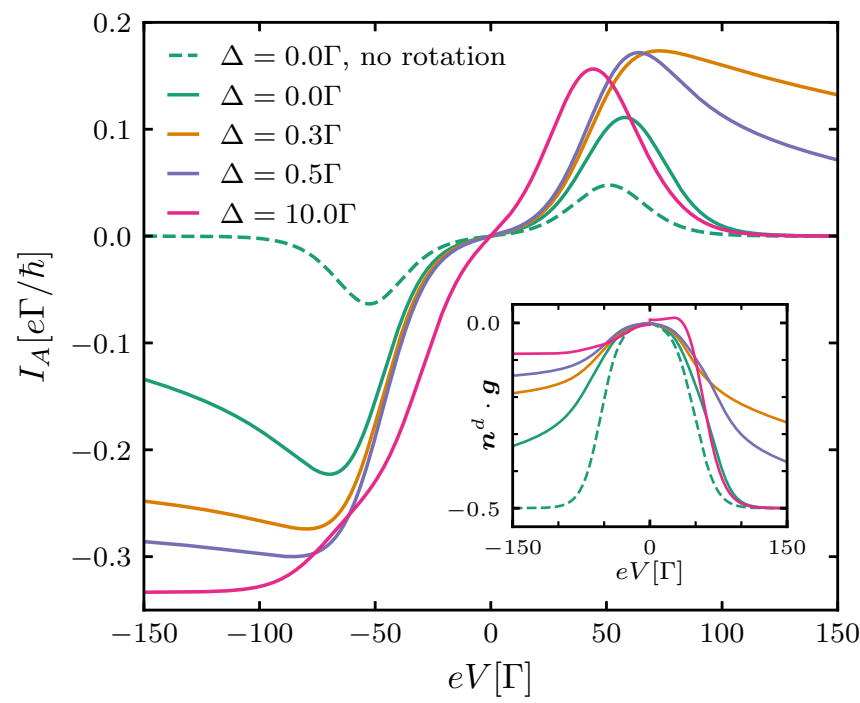

FIG. 2. Current-voltage characteristics for $\epsilon=25 \Gamma$ and detunings $\Delta_{13}=-\Delta_{23}=\Delta$ calculated with the flavor equations (4)-(10) (for $\Delta=0,0.3 \Gamma, 0.5 \Gamma$ ) and with Fermi's golden rule (for $\Delta=$ $10 \Gamma)$. For the dashed curve, the rotation term (8) has been omitted by hand. The inset shows the scalar product of drain and dot flavor polarization. Further parameters are $T=5 \Gamma$ and $\Lambda=1000 \Gamma$.

the order of $\Gamma$. For $V<0$, lead $B C$ is the drain electrode, $\boldsymbol{n}^{d}=\boldsymbol{n}^{B C}$. At high voltages and zero detuning, a full suppression of the current is obtained when omitting the rotation term (8) by hand (dashed line). In this case, the steady-state flavor polarization becomes $\boldsymbol{g}=(1,0,0,-1,0,-1,0,0) / \sqrt{3}$, which corresponds to the occupation of the dark state $\left|\psi_{123}\right\rangle=$ $(|1\rangle+|2\rangle-|3\rangle) / \sqrt{3}$ that decouples from the drain, i.e., the flavor-blockade conditions $P_{1}=1$ and $\boldsymbol{n}^{d} \cdot \boldsymbol{g}=-1 /(N-$ $1)=-1 / 2$ are satisfied. The blockade is partially lifted when the exchange-field- and detuning-induced flavor rotation is taken into account (see solid lines and inset), as they rotate the flavor polarization away from the blocking orientation. The magnitude of the exchange field falls off like $|\ln (|V| / 2 \Lambda)|$ at large voltages, which explains the observed NDC. Since away from resonance $\left|\mu_{r}-\epsilon\right| \gg T$ all Fermi functions are either 0 or 1, the voltage dependence of $\boldsymbol{B}_{\mathrm{ex}}$ is the sole cause of the NDC appearing here. While the perfect blockade in the absence of flavor rotation is not a generic feature, this reasoning actually applies to NDC in any multilevel-dot model: The exchange field rotates the flavor polarization into an orientation that increases $\boldsymbol{n}^{d} \cdot \boldsymbol{g}$, i.e., couples more strongly to the drain, and an NDC appears because $\left|\boldsymbol{B}_{\text {ex }}\right|$ decays with increasing voltage.

Returning to the model at hand, for large detuning (pink line), coherences are absent. This implies that flavor rotations vanish, but as the dark state $\left|\psi_{123}\right\rangle$ is a coherent superposition, it is not occupied to begin with and the current is not suppressed.

For $V>0$, lead $A$ becomes the drain electrode $\boldsymbol{n}^{d}=\boldsymbol{n}^{A}$. Our maximally symmetric model shows (nongeneric) striking current signatures here, which can easily be explained in the flavor framework. At zero detuning (green line) the flavor polarization is $g=(-\sqrt{3} / 2,0,0,0,0,0,0,1 / 2)$, which corresponds to the occupation of the dark state $\left|\psi_{12}\right\rangle=(|1\rangle-$ 
|2) $/ \sqrt{2}$ and satisfies the flavor-blockade conditions $P_{1}=1$ and $\boldsymbol{n}^{d} \cdot \boldsymbol{g}=-1 / 2$. In contrast to $V<0$, flavor rotations do not restore the current since they cannot affect the dark state, as $\boldsymbol{n}^{A} \wedge \boldsymbol{g}=0$ and $\boldsymbol{n}^{B C} \wedge \boldsymbol{g}=0$. This changes with small $|\Delta|$, where the flavor is rotated by the detuning-induced field $\boldsymbol{B}$. The resulting flavor is then affected by exchangefield-induced rotations and similarly to the case for $V<0$, off-resonance NDC appears because of the $V$ dependence of the exchange field. For large detuning, current is suppressed again since once an electron enters level 3 , it cannot leave anymore. However, compared to zero detuning, the physics involved is fundamentally different since the blockade can be understood in a simple Fermi golden rule approach.

\section{CONCLUSION}

We have introduced the concept of flavor polarization for the dynamics of $N$ quantum-dot levels in the coherentsequential-tunneling regime. The significance of the kinetic equations presented in this paper is threefold. First, they constitute a unifying description of multilevel quantum dots. Second, they allow for an intuitive interpretation of the dynamics in these systems in terms of accumulation, relaxation, and rotation of a flavor-polarization vector. Third, they isolate the entire bias-voltage dependence beyond the Fermi functions in a single term, the exchange field, which reveals flavor rotations as the origin of negative differential conductances in off-resonance regimes.

Our framework can straightforwardly be generalized to arbitrary occupations by introducing several dot flavor polarizations [45]. Furthermore, it will be also very useful for strong dot-lead coupling by taking higher-order tunneling processes into account using, e.g., real-time renormalizationgroup methods [46], where broadening and renormalization effects influence the resonance line shapes [47] and the Kondo effect occurs in the cotunneling regime [48-52].

\section{ACKNOWLEDGMENTS}

We thank R. Harlander, C. Lindner, and S. Siccha for fruitful discussions. This work was supported by the Deutsche Forschungsgemeinschaft via RTG 1995 and CRC 1242 (Project No. 278162697). Simulations were performed with computing resources granted by RWTH Aachen University under Project No. thes0595.

\section{APPENDIX A: DIAGRAMS}

The generalized transition matrix elements $W_{x x^{\prime}, \eta \eta^{\prime}}$ are represented as irreducible diagrams on the Keldysh contour. The

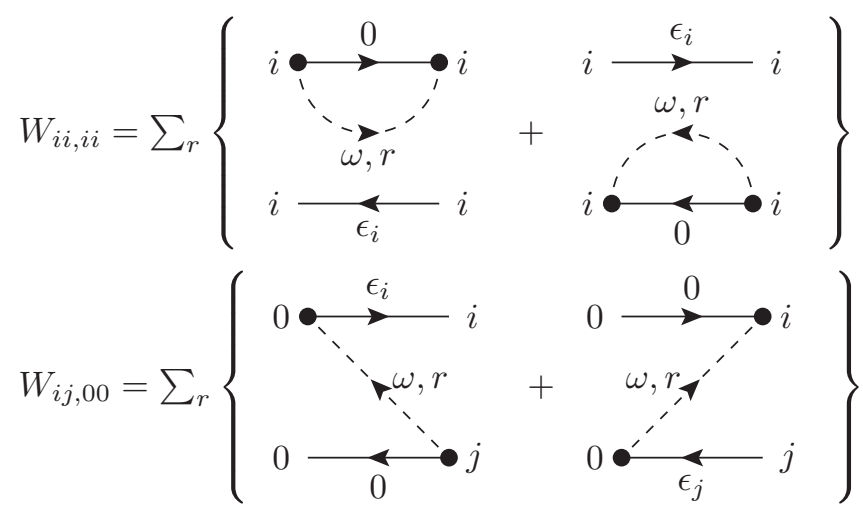

FIG. 3. Diagrams for two generalized transition matrix elements.

physical time axis runs from left to right, while the Keldysh contour runs from left to right and then back again. The rules for the evaluation of a diagram $W_{\chi \chi^{\prime}, \eta \eta^{\prime}}$ to first order in the tunneling strength $\Gamma$ are as follows.

(i) Draw all topologically different diagrams with states $\eta$ and $\eta^{\prime}$ to the left and $\chi$ and $\chi^{\prime}$ to the right. Assign dot states and their energies to all Keldysh contour elements between vertices representing the tunneling Hamiltonian. Vertices are connected in pairs by directed tunneling lines that carry a reservoir index $r$ and tunneling energy $\omega$. A first-order diagram contains one tunneling line connecting two vertices on the far left and far right of the diagram.

(ii) Each segment between vertices gives a factor $1 /(E+$ $i 0^{+}$), with $E$ being the difference of all energies going to the left minus all energies going to the right, including the tunneling line energy.

(iii) A tunneling line with index $r$ going from a vertex where a dot state $i$ is annihilated to a vertex where a dot state $j$ is created implies a factor $\Upsilon_{j i}^{r} \bar{\rho}_{r}(\omega) f_{r}^{ \pm}(\omega) / 2 \pi$, where $\bar{\rho}_{r}(\omega)=\rho_{r}(\omega) / \rho_{0}$ and $f_{r}^{+}(\omega)$ is to be taken if the line goes backward with respect to the Keldysh contour and $f_{r}^{-}(\omega)$ if it goes forward.

(iv) Assign a total prefactor $(-i)$ and for each vertex on the lower contour a prefactor -1 .

(v) Sum over internal indices and integrate over the tunneling energy $\omega$.

As an example, Fig. 3 shows the diagrams for two generalized transition matrix elements, with $i, j=1, \ldots, N$ labeling a dot level. According to the above rules, their values are in the limit of large $\Lambda$

$$
\begin{aligned}
W_{i i, i i} & =-i \sum_{r} \frac{\Upsilon_{i i}^{r}}{2 \pi} \int d \omega \bar{\rho}_{r}(\omega) f_{r}^{-}(\omega)\left(\frac{1}{\epsilon_{i}-\omega+i 0^{+}}+\frac{1}{\omega-\epsilon_{i}+i 0^{+}}\right)=-\sum_{r} \Upsilon_{i i}^{r} f_{r_{i}}^{-}\left(\epsilon_{i}\right), \\
W_{i j, 00} & =i \sum_{r} \frac{\Upsilon_{i j}^{r}}{2 \pi} \int d \omega \bar{\rho}_{r}(\omega) f_{r}^{+}(\omega)\left(\frac{1}{\omega-\epsilon_{i}+i 0^{+}}+\frac{1}{\epsilon_{j}-\omega+i 0^{+}}\right) \\
& =\sum_{r} \frac{\Upsilon_{i j}^{r}}{2}\left\{\left[f_{r}^{+}\left(\epsilon_{i}\right)+f_{r}^{+}\left(\epsilon_{j}\right)\right]+i\left[\Omega_{r}\left(\epsilon_{i}\right)-\Omega_{r}\left(\epsilon_{j}\right)\right]\right\},
\end{aligned}
$$


with $\Omega_{r}\left(\epsilon_{i}\right)=\frac{1}{\pi}\left[\operatorname{Re} \psi\left(\frac{1}{2}+i \frac{\beta}{2 \pi}\left(\mu_{r}-\epsilon_{i}\right)\right)-\psi\left(\frac{1}{2}+\frac{\beta \Lambda}{2 \pi}\right)\right]$, where $\psi$ is the digamma function.

The current into reservoir $r$ reads, to first order,

$$
I_{r}=\sum_{\chi \eta \eta^{\prime}} \sum_{m} m W_{\chi \chi, \eta \eta^{\prime}}^{r m} \rho_{\eta \eta^{\prime}} .
$$

Here $W_{\chi \chi, \eta \eta \eta^{\prime}}^{r m}$ are those first-order diagrams where the number of electrons entering reservoir $r$ minus those leaving reservoir $r$ is $m$.

\section{APPENDIX B: USEFUL RELATIONS FOR THE SU $(N)$ GENERATORS}

The generators $\lambda_{a}$ of $\mathrm{SU}(N)$ fulfill the relations

$$
\begin{aligned}
\operatorname{Tr}\left(\lambda_{a}\right) & =0, \\
\operatorname{Tr}\left(\lambda_{a} \lambda_{b}\right) & =2 \delta_{a b}, \\
{\left[\lambda_{a}, \lambda_{b}\right]_{-} } & =2 i \sum_{c} f_{a b c} \lambda_{c}, \\
{\left[\lambda_{a}, \lambda_{b}\right]_{+} } & =\frac{4}{N} \delta_{a b}+2 \sum_{c} d_{a b c} \lambda_{c} .
\end{aligned}
$$

We can express the antisymmetric tensors $f_{a b c}$ and $d_{a b c}$ as

$$
\begin{aligned}
d_{a b c} & =\frac{1}{4} \operatorname{Tr}\left(\left[\lambda_{a}, \lambda_{b}\right]_{+} \lambda_{c}\right), \\
f_{a b c} & =-\frac{i}{4} \operatorname{Tr}\left(\left[\lambda_{a}, \lambda_{b}\right]_{-} \lambda_{c}\right) .
\end{aligned}
$$

These relations will be used in the following proofs.

\section{APPENDIX C: SEMIPOSITIVITY OF THE RELAXATION MATRIX $D^{r}$}

The relaxation matrix $D^{r}$ is defined as

$$
D_{a c}^{r}=\delta_{a c}+c_{N} \sum_{b} d_{a b c} n_{b}^{r}
$$

or, equivalently,

$$
D^{r} \boldsymbol{g}=\boldsymbol{g}+\boldsymbol{n}^{r} * \boldsymbol{g} .
$$

We need to show that $D^{r}$ is positive semidefinite, i.e., $g$. $D^{r} \boldsymbol{g} \geqslant 0$ for any $\boldsymbol{g}$, to justify the interpretation of the corresponding term in the kinetic equation as a relaxation term. Using $\boldsymbol{g} \cdot \boldsymbol{g}=\sum_{a} g_{a} g_{a}$ and $\boldsymbol{g} \cdot\left(\boldsymbol{n}^{r} * \boldsymbol{g}\right)=c_{N} \sum_{a b c} d_{a b c} g_{a} n_{b}^{r} g_{c}=$ $c_{N} \sum_{a b c} d_{a b c} g_{a} g_{b} n_{c}^{r}$, we get

$$
\begin{aligned}
\boldsymbol{g} \cdot D^{r} \boldsymbol{g}= & \sum_{a}\left\{g_{a} g_{a}+c_{N} \sum_{b c} d_{a b c} g_{a} g_{b} n_{c}^{r}\right\} \\
= & \frac{1}{2} \sum_{a b} g_{a} g_{b} \operatorname{Tr}\left(\lambda_{a} \lambda_{b}\right) \\
& +\frac{c_{N}}{4} \sum_{a b c} \operatorname{Tr}\left(\left[\lambda_{a}, \lambda_{b}\right]_{+} \lambda_{c}\right) g_{a} g_{b} n_{c}^{r} \\
= & \frac{1}{2} \operatorname{Tr}\left[(\boldsymbol{g} \cdot \boldsymbol{\lambda})^{2}\right]+\frac{c_{N}}{4} \operatorname{Tr}\left([\boldsymbol{g} \cdot \boldsymbol{\lambda}, \boldsymbol{g} \cdot \boldsymbol{\lambda}]_{+} \boldsymbol{n}^{r} \cdot \boldsymbol{\lambda}\right) \\
= & \frac{1}{2} \operatorname{Tr}\left[(\boldsymbol{g} \cdot \boldsymbol{\lambda})^{2}\left(\mathbb{1}_{N}+c_{N} \boldsymbol{n}^{r} \cdot \boldsymbol{\lambda}\right)\right] .
\end{aligned}
$$

Since the hybridization matrix $\Upsilon^{r}=\Gamma^{r}\left(\mathbb{1}_{N}+c_{N} \boldsymbol{n}^{r} \cdot \lambda\right)$ is positive semidefinite and $\Gamma^{r}>0$, we can use the decomposition $\mathbb{1}_{N}+c_{N} \boldsymbol{n}^{r} \cdot \lambda=\sum_{i} \sigma_{i}|i\rangle\langle i|$, with $\sigma_{i} \geqslant 0$. This yields

$$
\begin{aligned}
\boldsymbol{g} \cdot D^{r} \boldsymbol{g} & =\frac{1}{2} \sum_{i j}\left\langle j\left|(\boldsymbol{g} \cdot \boldsymbol{\lambda})^{2} \sigma_{i}\right| i\right\rangle\langle i \mid j\rangle \\
& =\frac{1}{2} \sum_{j} \sigma_{j}\langle j|(\boldsymbol{g} \cdot \boldsymbol{\lambda})(\boldsymbol{g} \cdot \boldsymbol{\lambda})| j\rangle \\
& =\frac{1}{2} \sum_{j} \sigma_{j} \| \boldsymbol{g} \cdot \lambda|j\rangle \|^{2} \geqslant 0 .
\end{aligned}
$$

In the last line we have used the Hermiticity of $\lambda_{a}$ and $\sigma_{j} \geqslant 0$.

\section{APPENDIX D: SU(N) INVARIANCE}

Any $N \times N$ Hermitian matrix $M$ can be decomposed as $M=k \mathbb{1}_{N}+\boldsymbol{m} \cdot \boldsymbol{\lambda}$, with $k$ and $m_{a} \in \mathbb{R}$. After a basis change $M \rightarrow \tilde{M}=U M U^{\dagger}$, we can decompose similarly $\tilde{M}=k \mathbb{1}_{N}+$ $\tilde{\boldsymbol{m}} \cdot \lambda$. The elements of $\tilde{\boldsymbol{m}} \mathrm{read}$

$$
\begin{aligned}
\tilde{m}_{a} & =\operatorname{Tr}\left(\tilde{M} \lambda_{a}\right) / 2=\operatorname{Tr}\left(U M U^{\dagger} \lambda_{a}\right) / 2 \\
& =\sum_{b} m_{b} \operatorname{Tr}\left(U \lambda_{b} U^{\dagger} \lambda_{a}\right) / 2 \\
& =\sum_{b} R(U)_{a b} m_{b},
\end{aligned}
$$

or in matrix-vector notation $\tilde{\boldsymbol{m}}=R(U) \boldsymbol{m}$, where $R(U)$ is the $s_{N}$-dimensional rotation matrix corresponding to the basis transformation $U$.

The kinetic equations are written in terms of $P_{0}$ and $P_{1}$, which are obviously invariant under rotation, as well as $\boldsymbol{g}, \boldsymbol{n}^{\boldsymbol{r}}$, and $\boldsymbol{B}$, which transform as vectors. To prove the form invariance of the kinetic equation under an $\mathrm{SU}(N)$ transformation of the basis, we need to show that the scalar product $\boldsymbol{x} \cdot \boldsymbol{y}$ transforms like a scalar and the star and wedge products $\boldsymbol{x} * \boldsymbol{y}$ and $\boldsymbol{x} \wedge \boldsymbol{y}$ like vectors.

Let us start with the invariance of the scalar product

$$
\begin{aligned}
\boldsymbol{x} \cdot \boldsymbol{y} & =\sum_{a} x_{a} y_{a}=\frac{1}{2} \sum_{a b} x_{a} y_{b} \operatorname{Tr}\left(\lambda_{a} \lambda_{b}\right) \\
& =\frac{1}{2} \operatorname{Tr}[(\boldsymbol{x} \cdot \lambda)(\boldsymbol{y} \cdot \lambda)] \\
& =\frac{1}{2} \operatorname{Tr}\left[U(\boldsymbol{x} \cdot \boldsymbol{\lambda}) U^{\dagger} U(\boldsymbol{y} \cdot \boldsymbol{\lambda}) U^{\dagger}\right] \\
& =\frac{1}{2} \operatorname{Tr}[(\tilde{\boldsymbol{x}} \cdot \boldsymbol{\lambda})(\tilde{\boldsymbol{y}} \cdot \boldsymbol{\lambda})]=\tilde{\boldsymbol{x}} \cdot \tilde{\boldsymbol{y}} .
\end{aligned}
$$

Next we show the vector character of the star and wedge products by convincing ourselves that the combinations $(\boldsymbol{x} * \boldsymbol{y}) \cdot \boldsymbol{z}$ and $(\boldsymbol{x} \wedge \boldsymbol{y}) \cdot z$ remain invariant under rotation. We find

$$
\begin{aligned}
(\boldsymbol{x} * \boldsymbol{y}) \cdot \boldsymbol{z} & =c_{N} \sum_{a b c} d_{a b c} x_{a} y_{b} z_{c} \\
& =\frac{c_{N}}{4} \sum_{a b c} \operatorname{Tr}\left(\left[\lambda_{a}, \lambda_{b}\right]_{+} \lambda_{c}\right) x_{a} y_{b} z_{c} \\
& =\frac{c_{N}}{4} \operatorname{Tr}\left\{[(\boldsymbol{x} \cdot \boldsymbol{\lambda}),(\boldsymbol{y} \cdot \boldsymbol{\lambda})]_{+}(\boldsymbol{z} \cdot \boldsymbol{\lambda})\right\}
\end{aligned}
$$




$$
\begin{aligned}
& =\frac{c_{N}}{4} \operatorname{Tr}\left\{[(\tilde{\boldsymbol{x}} \cdot \lambda),(\tilde{\boldsymbol{y}} \cdot \boldsymbol{\lambda})]_{+}(\tilde{\boldsymbol{z}} \cdot \boldsymbol{\lambda})\right\} \\
& =(\tilde{\boldsymbol{x}} * \tilde{\boldsymbol{y}}) \cdot \tilde{z}
\end{aligned}
$$

as well as

$$
\begin{aligned}
(\boldsymbol{x} \wedge \boldsymbol{y}) \cdot \boldsymbol{z} & =c_{N} \sum_{a b c} f_{a b c} x_{a} y_{b} z_{c} \\
& =-i \frac{c_{N}}{4} \sum_{a b c} \operatorname{Tr}\left(\left[\lambda_{a}, \lambda_{b}\right]_{-} \lambda_{c}\right) x_{a} y_{b} z_{c} \\
& =-i \frac{c_{N}}{4} \operatorname{Tr}\left\{[(\boldsymbol{x} \cdot \boldsymbol{\lambda}),(\boldsymbol{y} \cdot \boldsymbol{\lambda})]_{-}(\boldsymbol{z} \cdot \boldsymbol{\lambda})\right\}
\end{aligned}
$$

$$
\begin{aligned}
& =-i \frac{c_{N}}{4} \operatorname{Tr}\left\{[(\tilde{\boldsymbol{x}} \cdot \boldsymbol{\lambda}),(\tilde{\boldsymbol{y}} \cdot \boldsymbol{\lambda})]_{-}(\tilde{\boldsymbol{z}} \cdot \boldsymbol{\lambda})\right\} \\
& =(\tilde{\boldsymbol{x}} \wedge \tilde{\boldsymbol{y}}) \cdot \tilde{z}
\end{aligned}
$$

which completes the proof of the $\mathrm{SU}(N)$ invariance of the kinetic equations.

\section{APPENDIX E: EXPLICIT FORM OF THE GELL-MANN MATRICES}

In the example of the triple quantum dot, we choose the standard Gell-Mann matrices for expressing the flavorpolarization vectors. These are given by

$$
\begin{array}{lll}
\lambda_{1}=\left(\begin{array}{lll}
0 & 1 & 0 \\
1 & 0 & 0 \\
0 & 0 & 0
\end{array}\right), & \lambda_{2}=\left(\begin{array}{ccc}
0 & -i & 0 \\
i & 0 & 0 \\
0 & 0 & 0
\end{array}\right), & \lambda_{3}=\left(\begin{array}{ccc}
1 & 0 & 0 \\
0 & -1 & 0 \\
0 & 0 & 0
\end{array}\right), \\
\lambda_{4}=\left(\begin{array}{lll}
0 & 0 & 1 \\
0 & 0 & 0 \\
1 & 0 & 0
\end{array}\right), & \lambda_{5}=\left(\begin{array}{ccc}
0 & 0 & -i \\
0 & 0 & 0 \\
i & 0 & 0
\end{array}\right), & \\
\lambda_{6}=\left(\begin{array}{lll}
0 & 0 & 0 \\
0 & 0 & 1 \\
0 & 1 & 0
\end{array}\right), & \lambda_{7}=\left(\begin{array}{ccc}
0 & 0 & 0 \\
0 & 0 & -i \\
0 & i & 0
\end{array}\right), & \lambda_{8}=\frac{1}{\sqrt{3}}\left(\begin{array}{ccc}
1 & 0 & 0 \\
0 & 1 & 0 \\
0 & 0 & -2
\end{array}\right) .
\end{array}
$$

[1] J. König and J. Martinek, Interaction-Driven Spin Precession in Quantum-Dot Spin Valves, Phys. Rev. Lett. 90, 166602 (2003).

[2] M. Braun, J. König, and J. Martinek, Theory of transport through quantum-dot spin valves in the weak-coupling regime, Phys. Rev. B 70, 195345 (2004).

[3] S. Braig and P. W. Brouwer, Rate equations for Coulomb blockade with ferromagnetic leads, Phys. Rev. B 71, 195324 (2005).

[4] W. Rudziński, J. Barnaś, R. Świrkowicz, and M. Wilczyński, Spin effects in electron tunneling through a quantum dot coupled to noncollinearly polarized ferromagnetic leads, Phys. Rev. B 71, 205307 (2005).

[5] I. Weymann, J. König, J. Martinek, J. Barnaś, and G. Schön, Tunnel magnetoresistance of quantum dots coupled to ferromagnetic leads in the sequential and cotunneling regimes, Phys. Rev. B 72, 115334 (2005).

[6] R. Hornberger, S. Koller, G. Begemann, A. Donarini, and M. Grifoni, Transport through a double-quantum-dot system with noncollinearly polarized leads, Phys. Rev. B 77, 245313 (2008).

[7] M. Hell, B. Sothmann, M. Leijnse, M. R. Wegewijs, and J. König, Spin resonance without spin splitting, Phys. Rev. B 91, 195404 (2015).

[8] N. M. Gergs, S. A. Bender, R. A. Duine, and D. Schuricht, Spin Switching via Quantum Dot Spin Valves, Phys. Rev. Lett. 120, 017701 (2018).

[9] L. Y. Zhang, C. Y. Wang, Y. G. Wei, X. Y. Liu, and D. Davidović, Spin-polarized electron transport through nanometer-scale Al grains, Phys. Rev. B 72, 155445 (2005).

[10] K. Hamaya, M. Kitabatake, K. Shibata, M. Jung, M. Kawamura, K. Hirakawa, T. Machida, T. Taniyama, S. Ishida, and Y.
Arakawa, Electric-field control of tunneling magnetoresistance effect in a Ni/InAs/Ni quantum-dot spin valve, Appl. Phys. Lett. 91, 022107 (2007).

[11] A. Crisan, S. Datta, J. Viennot, M. Delbecq, A. Cottet, and T. Kontos, Harnessing spin precession with dissipation, Nat. Commun. 7, 10451 (2016).

[12] D. Xiao, W. Yao, and Q. Niu, Valley-Contrasting Physics in Graphene: Magnetic Moment and Topological Transport, Phys. Rev. Lett. 99, 236809 (2007).

[13] J. R. Schaibley, H. Yu, G. Clark, P. Rivera, J. S. Ross, K. L. Seyler, W. Yao, and X. Xu, Valleytronics in 2D materials, Nat. Rev. Mater. 1, 16055 (2016).

[14] A. W. Holleitner, C. R. Decker, H. Qin, K. Eberl, and R. H. Blick, Coherent Coupling of Two Quantum Dots Embedded in an Aharonov-Bohm Interferometer, Phys. Rev. Lett. 87, 256802 (2001).

[15] J. König and Y. Gefen, Aharonov-Bohm interferometry with interacting quantum dots: Spin configurations, asymmetric interference patterns, bias-voltage-induced Aharonov-Bohm oscillations, and symmetries of transport coefficients, Phys. Rev. B 65, 045316 (2002).

[16] T. Hatano, T. Kubo, Y. Tokura, S. Amaha, S. Teraoka, and S. Tarucha, Aharonov-Bohm Oscillations Changed by Indirect Interdot Tunneling via Electrodes in Parallel-Coupled Vertical Double Quantum Dots, Phys. Rev. Lett. 106, 076801 (2011).

[17] M. Governale, M. G. Pala, and J. König, Real-time diagrammatic approach to transport through interacting quantum dots with normal and superconducting leads, Phys. Rev. B 77, 134513 (2008). 
[18] A. Vidan, R. M. Westervelt, M. Stopa, M. Hanson, and A. C. Gossard, Triple quantum dot charging rectifier, Appl. Phys. Lett. 85, 3602 (2004).

[19] L. Gaudreau, S. A. Studenikin, A. S. Sachrajda, P. Zawadzki, A. Kam, J. Lapointe, M. Korkusinski, and P. Hawrylak, Stability Diagram of a Few-Electron Triple Dot, Phys. Rev. Lett. 97, 036807 (2006).

[20] D. Schröer, A. D. Greentree, L. Gaudreau, K. Eberl, L. C. L. Hollenberg, J. P. Kotthaus, and S. Ludwig, Electrostatically defined serial triple quantum dot charged with few electrons, Phys. Rev. B 76, 075306 (2007).

[21] M. C. Rogge and R. J. Haug, Two-path transport measurements on a triple quantum dot, Phys. Rev. B 77, 193306 (2008).

[22] M. H. Hettler, W. Wenzel, M. R. Wegewijs, and H. Schoeller, Current Collapse in Tunneling Transport through Benzene, Phys. Rev. Lett. 90, 076805 (2003).

[23] D. Darau, G. Begemann, A. Donarini, and M. Grifoni, Interference effects on the transport characteristics of a benzene single-electron transistor, Phys. Rev. B 79, 235404 (2009).

[24] R. Alicki and K. Lendi, Quantum Dynamical Semigroups and Applications, Lecture Notes in Physics Vol. 717 (Springer, Berlin, 2007).

[25] F. T. Hioe and J. H. Eberly, $N$-Level Coherence Vector and Higher Conservation Laws in Quantum Optics and Quantum Mechanics, Phys. Rev. Lett. 47, 838 (1981).

[26] M. S. Byrd and N. Khaneja, Characterization of the positivity of the density matrix in terms of the coherence vector representation, Phys. Rev. A 68, 062322 (2003).

[27] G. Kimura, The Bloch vector for $N$-level systems, Phys. Lett. A 314, 339 (2003).

[28] L. Jakóbczyk and M. Siennicki, Geometry of Bloch vectors in two-qubit system, Phys. Lett. A 286, 383 (2001).

[29] J. König, J. Schmid, H. Schoeller, and G. Schön, Resonant tunneling through ultrasmall quantum dots: Zero-bias anomalies, magnetic-field dependence, and boson-assisted transport, Phys. Rev. B 54, 16820 (1996).

[30] J. König, H. Schoeller, and G. Schön, Zero-Bias Anomalies and Boson-Assisted Tunneling Through Quantum Dots, Phys. Rev. Lett. 76, 1715 (1996).

[31] K. S. Mallesh and N. Mukunda, The algebra and geometry of SU(3) matrices, Pramana 49, 371 (1997).

[32] J. Chen, M. A. Reed, A. M. Rawlett, and J. M. Tour, Large on-off ratios and negative differential resistance in a molecular electronic device, Science 286, 1550 (1999).

[33] B. Michaelis, C. Emary, and C. W. J. Beenakker, All-electronic coherent population trapping in quantum dots, Europhys. Lett. 73, 677 (2006).

[34] C. Emary, Dark states in the magnetotransport through triple quantum dots, Phys. Rev. B 76, 245319 (2007).

[35] C. Payette, G. Yu, J. A. Gupta, D. G. Austing, S. V. Nair, B. Partoens, S. Amaha, and S. Tarucha, Coherent Three-Level Mixing in an Electronic Quantum Dot, Phys. Rev. Lett. 102, 026808 (2009).

[36] C. Pöltl, C. Emary, and T. Brandes, Two-particle dark state in the transport through a triple quantum dot, Phys. Rev. B 80, 115313 (2009).
[37] M. Busl, R. Sánchez, and G. Platero, Control of spin blockade by ac magnetic fields in triple quantum dots, Phys. Rev. B 81, 121306(R) (2010).

[38] A. Donarini, G. Begemann, and M. Grifoni, Interference effects in the Coulomb blockade regime: Current blocking and spin preparation in symmetric nanojunctions, Phys. Rev. B 82, 125451 (2010).

[39] I. Weymann, B. R. Bułka, and J. Barnaś, Dark states in transport through triple quantum dots: The role of cotunneling, Phys. Rev. B 83, 195302 (2011).

[40] B. Xu and Y. Dubi, Negative differential conductance in molecular junctions: An overview of experiment and theory, J. Phys. Condens. Matter 27, 263202 (2015).

[41] K. Wrześniewski and I. Weymann, Dark states in spin-polarized transport through triple quantum dot molecules, Phys. Rev. B 97, 075425 (2018).

[42] A. Donarini, M. Niklas, M. Schafberger, N. Paradiso, C. Strunk, and M. Grifoni, Coherent population trapping by dark state formation in a carbon nanotube quantum dot, Nat. Commun. 10, 381 (2019).

[43] M. Niklas, A. Trottmann, A. Donarini, and M. Grifoni, Fano stability diagram of a symmetric triple quantum dot, Phys. Rev. B 95, 115133 (2017).

[44] M. Gell-Mann, Symmetries of baryons and mesons, Phys. Rev. 125, 1067 (1962).

[45] M. T. Maurer, J. König, and H. Schoeller (unpublished).

[46] H. Schoeller, Dynamics of open quantum systems, in Computing Solids: Models, Ab-Initio Methods and Supercomputing, edited by S. Blügel, N. Helbig, V. Meden, and D. Wortmann, Lecture Notes of the 45th IFF Spring School 2014, Vol. 74 (Forschungszentrum Jülich GmbH, Jülich, 2014).

[47] C. J. Lindner, F. B. Kugler, V. Meden, and H. Schoeller, Renormalization group transport theory for open quantum systems: Charge fluctuations in multilevel quantum dots in and out of equilibrium, Phys. Rev. B 99, 205142 (2019).

[48] S. Göttel, F. Reininghaus, and H. Schoeller, Generic fixed point model for pseudo-spin- $\frac{1}{2}$ quantum dots in nonequilibrium: Spin-valve systems with compensating spin polarizations, Phys. Rev. B 92, 041103(R) (2015).

[49] R. López, T. Rejec, J. Martinek, and R. Žitko, SU(3) Kondo effect in spinless triple quantum dots, Phys. Rev. B 87, 035135 (2013).

[50] C. J. Lindner, F. B. Kugler, H. Schoeller, and J. von Delft, Flavor fluctuations in three-level quantum dots: Generic SU(3) Kondo fixed point in equilibrium and non-Kondo fixed points in nonequilibrium, Phys. Rev. B 97, 235450 (2018).

[51] M. Arnold, T. Langenbruch, and J. Kroha, Stable Two-Channel Kondo Fixed Point of an SU(3) Quantum Defect in a Metal: Renormalization-Group Analysis and Conductance Spikes, Phys. Rev. Lett. 99, 186601 (2007).

[52] J. Paaske, A. Rosch, P. Wölfle, N. Mason, C. M. Marcus, and J. Nygård, Non-equilibrium singlet-triplet Kondo effect in carbon nanotubes, Nat. Phys. 2, 460 (2006). 